\title{
Binaural integration of melodic patterns
}

\author{
DIANA DEUTSCH \\ Center for Human Information Processing, University of California at San Diego \\ La Jolla, California 92093
}

\begin{abstract}
It has been argued that there is a limit to the rate at which we can switch attention between ears in monitoring auditory information. Listeners identified melodic configurations formed by rapid sequences of tones. When these sequences were presented binaurally, excellent performance was obtained. Yet when the component tones of the melody were distributed between the ears, performance was largely nullified when a drone (i.e., a lower constantfrequency tone) was presented to the ear opposite that receiving the melody component. This improvement in performance cannot be attributed to processing the harmonic relationships between melody and drone, since when, instead, the drone was presented to the same ear as the melody component, performance was at chance. Onset-offset asynchronies between the drone and melody components resulted in performance levels between those where the drone and melody components were synchronous and those where the melody switched between ears without an accompanying drone. It is argued that difficulties in binaural integration are due not to processing limitations, but to a mechanism that is invoked under certain conditions to prevent confusion in monitoring individual sound sources.
\end{abstract}

It has been argued on a number of grounds that we have difficulty in integrating rapid streams of acoustic information when these are presented separately to the two ears. For instance, Cherry and Taylor (1954) studied the intelligibility of speech which was switched alternately between the ears, and found a substantial drop in intelligibility at alternation rates of around $3 \mathrm{cps}$. They interpreted their results in terms of a limitation in the rate at which we can switch our attention from ear to ear. This explanation was, however, challenged by Huggins (1964), who found that the maximum dip in intelligibility shifted in parallel with a shift in the speech rate. He therefore argued that this effect was due to interference in the processing of basic units of speech, rather than to a limitation in attention switching time.

A second line of evidence involves the recall of lists of dichotically presented digits. Broadbent (1954, 1958) found that when two lists of digits are simultaneously presented at fast rates, one to each ear, subjects recall these better by ear than by temporal order, which would require switching between ears. Treisman (1971) further found that subjects were less able to recall successive lists of digits when these were presented alternately to the two ears than when they were presented binaurally. These effects cannot be ascribed to a perceptual interference with basic

This work was supported by United States Public Health Service Grant MH-21001. I am especially indebted to R. Erickson for valuable discussions concerning channeling principles in music. Requests for reprints should be sent to Diana Deutsch, Department of Psychology, C-009, University of California at San Diego, La Jolla, California 92093. units of speech, since the integrity of the verbal items was preserved in these experiments.

A further argument was advanced by Axelrod, Guzy, and Diamond (1968). They required subjects to compare the repetition rates of clicks which were presented either monotically or alternating dichotically, and found that the dichotic rates were consistently underestimated relative to the monotic one, the degree of this underestimation increasing with increasing repetition rate. The authors explain this effect as due to an inability to attend in rapid alternation to the inputs to the two ears, this resulting in a loss of information in the dichotic condition.

However, arguments may be advanced against the notion of a fixed limitation in the rate at which we can monitor events at the two ears. Perhaps the most compelling argument arises from general observation. In normal listening, the information arriving at our two ears is never identical; and the running cross-correlations performed on this information are very important for a number of functions. One such function is auditory localization, and another is the suppression of echoes and reverberation (Haas, 1951; Tobias \& Schubert, 1959; Wallach, Newman, \& Rosenzweig, 1949). The acoustic elements that are compared here may be separated by no more than a few microseconds. It is difficult to reconcile such perceptual phenomena with the notion of a slow switching mechanism.

Furthermore, experiments using different paradigms have provided evidence against this hypothesis. Egan and Benson (1966) found that detection of tones in noise was not affected by advance knowledge of the target tone. Sorkin, Pastore, and Pohlman (1972) 
and Sorkin, Pohlman and Gilliom (1973) have shown that signal detection performance is not influenced by whether attention is focused on one ear or distributed across the ears, provided that the signals are nonsimultaneous. Decrements only occur under certain simultaneous conditions; and these could be based on several factors, such as perceptual distortion or a difficulty in localizing simultaneous signals (see also Ahroon, Pastore, \& Wolz, 1977, and Puleo and Pastore, in press). Moray (1975) also concluded, from experiments involving dichotic tone detections, that the two ears act essentially as independent processers. Using verbal stimuli, Shiffrin, Pisoni, and CastanedaMendez (1974) found that perception of stop consonants was unaffected by prior knowledge of ear of input, and also concluded against the notion of a limitation in switching rate at the perceptual level. Pollack (1978) attacked the question from a different point of view. He presented random polaritymodulated pulse trains to the two ears with controlled periods of interaural phase agreement and disagreement. He found that discrimination suffered only at switching rates above $2-3 \mathrm{msec}$, which closely matches the limitation in integration time found in certain nonaural tasks. Pollack therefore concluded that this limit was due to processes other than attention switching between the ears.

We are therefore faced with two conflicting sets of phenomena, one arguing for a decrement in processing information where rapid switching between the ears is involved and the other arguing against such a decrement. These conflicting phenomena lend themselves to the following interpretation. In our natural environment, signals arriving simultaneously from different sources are superimposed on each other before reaching our ears. One important function performed by our auditory system is to identify these sources and to separate out the signals emitted by each of them. If such perceptual separations were not accomplished, we would not know which elements of the acoustic spectrum to link with which so as to form higher-order abstractions. That is, if all firstorder acoustic elements were indiscriminately linked together, auditory shape recognition operations could not be performed. It is necessary, therefore, that there be a mechanism which inhibits the formation of higher-order linkages between acoustic elements which are likely to be emanating from different sources. Given the complexity of the acoustic environment (for instance the existence of echoes and reverberation), such a mechanism must be flexible and must use multiple criteria. Thus, certain stimulus configurations involving input to the two ears would be interpreted as emanating from the same source, so that integration of this information should be easy. Yet other stimulus configurations would be interpreted as emanating from two separate sources, so that in- tegration would be difficult. Thus, according to this hypothesis, when a decrement in integrating input to the two ears occurs, this is due not to capacity limitation, but rather to a mechanism which is imposed to prevent confusion. [An analogous argument has been advanced by Bregman (1978) to explain stream segregation phenomena in a variety of monaural tasks.]

The present experiments provide evidence for this hypothesis. They deal with the perceptual integration of rapid melodic patterns when the component tones are switched from ear to ear. Given certain background conditions, perceptual integration of such melodic patterns is extremely difficult. Yet, given other background conditions, these same patterns are readily integrated. It is argued that, in the former case, the total stimulus configuration is such that most probably the component tones would be emanating from two separate sound sources and that, in the latter case, it is more probable that the component tones would be emanating from a single source.

The factor manipulated here to produce these different processing strategies was the temporal relationship between the signals arriving at the two ears. Given the complexity of the acoustic environment (Benade, 1976) when a sound mixture is presented such that one ear receives one portion and the other ear receives a different portion, it is unclear which elements of the total spectrum should be assigned to one source and which to another, or, indeed, whether two sources rather than one are involved. If the onsets of these two signals are strictly synchronous, then, by the "law of common fate," this is evidence that a single source is involved. When a succession of such simultaneous signals is presented, factors such as frequency proximity between successive components of these spectra become important as clues concerning the external sources and their emissions (Deutsch, $1975 \mathrm{a}, 1975 \mathrm{~b})$. However, if the two ears are not presented with sounds simultaneously, but the signals arriving at each ear are clearly separated in time, this ambiguity of interpretation disappears, and inferences concerning sources can be made simply on the basis of ear of input.

\section{EXPERIMENT 1}

In the first experiment, subjects were required to identify melodic patterns when the component tones switched between the ears. Conditions were compared where input was to one ear at a time and where input was to the two ears simultaneously. Such simultaneity was achieved by presenting a drone (i.e., a lower constant-frequency tone) to the ear opposite that receiving a component of the melody. To control for the effect of the drone apart from its providing a simultaneous signal to the opposite ear, a third condition was added where the drone was presented to 

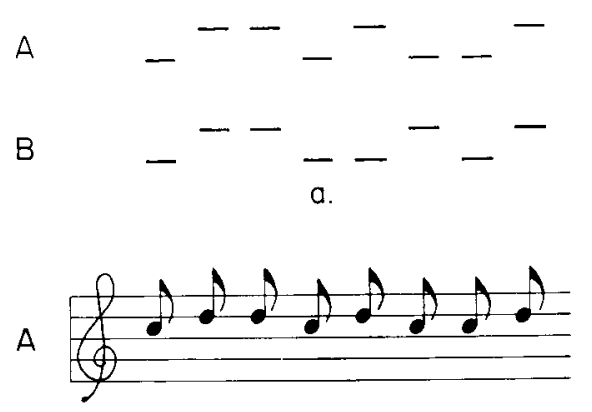

B

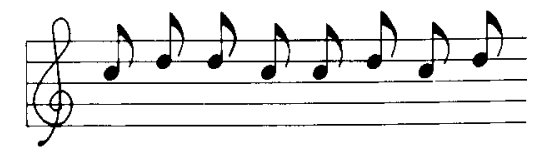

b.

Figure 1. Basic melodic patterns employed in Experiments 1 and 2 . (a) The patterns in diagram form, as displayed to subjects. (b) The patterns in musical notation.

the same ear as that receiving the component of the melody. In a fourth condition, the melody was presented binaurally.

It was predicted that integration of the melodic patterns would be very difficult in the nonsimultaneous conditions (i.e., where input was to only one ear at a time) and much easier in the simultaneous conditions (where either the melody was binaurally presented or a drone was presented to the opposite ear.)

\section{Method}

\section{Procedure}

On each trial, subjects were presented with a sequence of tones which consisted of 10 repetitions of a basic 8-tone pattern. Two such patterns were employed, and on each trial subjects identified, on forced choice, which of these had been presented. They indicated their judgments by writing " $A$ " or " $B$ " on paper. The answer sheets contained illustrations of Patterns $A$ and $B$, as shown in Figure 1a. Before the experiment began, the subjects were familiarized with these two sequences presented through loudspeakers.

\section{Conditions}

Examples of the two basic melodic patterns are shown in Figures $1 \mathrm{a}$ and $1 \mathrm{~b}$. Sequences composed of these patterns were presented under four experimental conditions.

Condition 1. All tones were presented simultaneously to both ears. Four examples of Pattern A were presented and four examples of Pattern B.

Condition 2. The component tones of the basic pattern were distributed in quasi-random fashion between the ears. An example of such a distribution is shown on Figure 2. Four such distributions were constructed. In each of these, four of the eight tones were presented to the right ear and four to the left; no more than three tones were presented consecutively to any one ear. For each sequence, the 10 repetitions of the basic pattern were presented with the component tones distributed in identical fashion between the ears. Sequences consisting of Patterns A and $B$ were presented once in each of these four configurations.

Condition 3. This condition was identical to Condition 2, except that whenever a component of the melody was presented to the right ear, a drone was simultaneously presented to the left ear; and whenever a component of the melody was presented to the left ear, a drone was simultaneously presented to the right ear. An example of such a distribution is shown on Figure 2. The drone was presented in exact synchrony with the component of the melody.

Condition 4. This condition was identical to Condition 3, except that here the drone was always presented to the same ear as that receiving the component of the melody rather than to the opposite ear. An example of such a distribution is shown on Figure 2.

The sequences were presented in random order in two blocks of 16 trials. The entire set of sequences was presented in two consecutive sessions, and the results were averaged.

\section{Stimulus Parameters}

All tones were $30 \mathrm{msec}$ in duration, and tones within a sequence were separated by $100-\mathrm{msec}$ pauses. The frequencies comprising the melodic patterns were 517 and $581 \mathrm{~Hz}\left(C_{5}\right.$ and $D_{5}$ on the equal-tempered scale; International Pitch; $\mathrm{A}=435$ ). When a drone was presented, this was always $345 \mathrm{~Hz}\left(\mathrm{~F}_{4}\right.$ on the same scale) (Figure 2). All trials within blocks were separated by an intertrial interval of $10 \mathrm{sec}$ duration, with 2-min pauses between blocks. As a warning signal, a $500-\mathrm{msec}$ tone of $2,000 \mathrm{~Hz}$ was presented $15 \mathrm{sec}$ before the onset of a block of trials.

\section{Apparatus}

Tones were generated by two Wavetek function generators (Model 155) controlled by a PDP-8/L computer, and were recorded on tape. The taped stimuli were low-pass filtered through two Krohn-Hite filters set at $1,500 \mathrm{~Hz}$; then through a Crown amplifier, and presented to subjects through Grason-Stadler TDH49 headphones, calibrated and matched, at a level of $75 \mathrm{~dB}$ SPL. The subjects were seated in sound-insulated booths.

\section{Subjects}

Twelve undergraduates at the University of California at San Diego served as subjects in this experiment. The subjects were paid

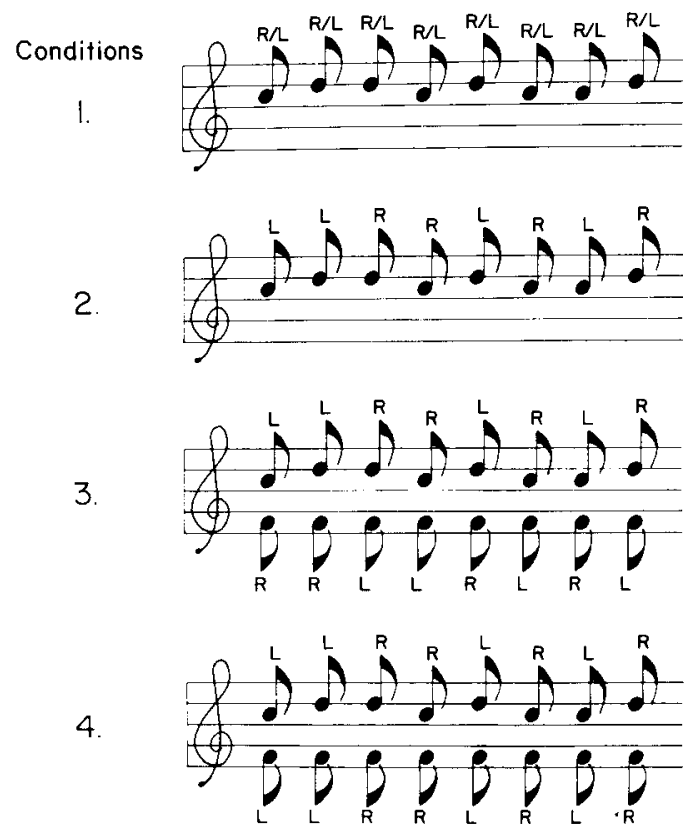

Figure 2. Examples of distributions between ears of melodic pattern and drone in the different conditions of Experiment 1. See text for details. 
for their services. They had all participated in experiments on memory for tonal pitch and were known, on this basis, to be excellent listeners. The subjects were further selected on the basis of being able to discriminate sequences consisting of Patterns A or B with ease when these were presented through loudspeakers.

\section{Results}

The percentage correct recognitions of the melodic patterns in the different conditions of the experiment are shown on Table 1 . It can be seen that performance on Condition 1 , where the tones were binaurally presented, was excellent. This is as expected, given the criterion used for subject selection. However, performance in Condition 2, where the component tones of the melody were distributed between the ears, was very poor. Thus, the procedure of switching this information from ear to ear produced a considerable decrement in identification performance. Yet, in Condition 3, where a drone was presented to the ear opposite that receiving a component of the melody, the performance level was again very high. This result cannot be attributed to processing the harmonic relationships between the drone and the components of the melody, since, in Condition 4 , where the drone was presented to the same ear as that receiving a component of the melody, performance was below chance.

Highly significant differences were found on comparing conditions where presentation was nonsimultaneous with the binaural condition (Condition 1 vs. Condition 2; Condition 1 vs. Condition 4 ; p < .01 , two-tailed, on Wilcoxon tests for both comparisons) and also on comparing the nonsimultaneous conditions with the condition where a drone was presented to the opposite ear (Condition 3 vs. Condition 2; Condition 3 vs. Condition 4; $p<.01$, two-tailed, on Wilcoxon tests for both comparisons).

It was also found that, on comparing both the two simultaneous conditions and the two nonsimultaneous conditions, performance level was significantly lower in the presence of the drone (Condition 1 vs. Condition 3; Condition 2 vs. Condition 4 ; $p<.05$. twotailed, for both comparisons). Thus the overall effect of the drone itself was one of slight interference.

\section{Discussion}

The results of this experiment clearly support the hypothesis. A marked decrement in melody identification occurred when the components of the melody switched between the ears, in accordance with findings such as those of Broadbent $(1954,1958)$, Cherry and Taylor (1954), and Treisman (1971), cited earlier. However, this decrement was largely nullified when a drone was presented to the ear opposite that receiving the melody component. Thus, the difficulty in integrating the melody across ears could not be
Table 1

Percent Average Error in the Different Conditions of Experiment 1

\begin{tabular}{lr}
\hline \multicolumn{1}{c}{ Condition } & $\begin{array}{c}\text { Error } \\
\text { Rate }\end{array}$ \\
\hline $\begin{array}{l}\text { (1) Melody binaural; no drone. } \\
\text { (2) Component tones of melody switch between ears; } \\
\text { no drone. }\end{array}$ & 5.7 \\
$\begin{array}{l}\text { (3) Component tones of melody switch between ears; } \\
\text { drone presented in synchrony to opposite ear. }\end{array}$ & 40.1 \\
$\begin{array}{l}\text { (4) Component tones of melody switch between ears; } \\
\text { drone presented in synchrony to same ear. }\end{array}$ & 16.1 \\
\hline
\end{tabular}

due to a limitation in the rate at which we can switch our attention from ear to ear, but rather to a mechanism which is invoked given the total stimulus configuration.

It should be noted that the melody components employed here were two semitones apart in pitch, and so safely within the range where they would be integrated into a single perceptual stream in binaural listening (Bregman, 1978; Bregman \& Campbell, 1971; Dowling, 1973; Van Noorden, 1975). However, the drone was seven semitones below the lowest melody component. Thus, if frequency proximity were the organizing principle for this configuration, we would expect the formation of two streams, the first comprising the melody components and the second the drone. This did, in fact, occur in the condition where the two ears received input simultaneously, but not where the drone and the melody components were delivered to the same ear. So we have here a situation where two organizing principles are set in competition with each other, the first based on frequency proximity and the second on spatial location. Where input is to one ear at a time, localization cues are very compelling, so that linkages are formed on the basis of ear of input and not frequency proximity. However, when both ears receive input simultaneously, an ambituity arises as to the sources of these inputs, so that organization by frequency proximity becomes a more reasonable principle.

Recently, Judd (in press) independently performed an experiment which was closely related to the present one. He presented subjects with four-tone melodic patterns whose components alternated from ear to ear, and found that presenting noise to the ear contralateral to the ear receiving the melody component resulted in improved recognition performance. $\mathrm{He}$ also advanced an interpretation in terms of competing channeling principles, arguing that the strong localization cues present in the no-noise condition induced a channeling by spatial location, and the weaker localization cues due to the noise resulted in a channeling instead by frequency proximity.

A result obtained by Schubert and Parker (1956) may also have a similar interpretation. They measured 
the amount of interference in speech perception produced by switching the signal from ear to ear, and found that adding noise to the contralateral ear reduced this interference effect. We may argue that under such simultaneous conditions the complex signal is interpreted in terms of two sources, one emitting noise and the other speech.

In the present experiment, mislocalization effects were also noted in the presence of a contralateral drone. These effects were highly idiosynchratic to the subject. For instance, some perceived the melody as localized on one side and the drone as localized on the other; others perceived the drone as in the center of the head and the melody as slowly shifting its position. It is assumed that these mislocalization effects were due to the same mechanism as permitted integration of the melody across ears, i.e., the interpretation of the melody components as emanating from one source and the drone as from another. A similar explanation was advanced by Deutsch (1975b) for mislocalization effects in the scale illusion.

A related mislocalization effect was described by Warren and Bashford (1976) and termed "auditory contralateral induction." They presented signals (tones or filtered speech) to one ear and noise to the other, and reversed the sides receiving the signal and noise every $1 / 2 \mathrm{sec}$. When the noise contained spectral components of the signal at appropriate intensity levels, the noise appeared to alternate from side to side, while the signal appeared stationary and diffusely localized about the midsaggital plane. The authors interpret the effect in the following way. In everyday life, a noise in one spatial location could cause an asymmetrical masking of a signal originating in another location, with the result that localization to the unmasked side would occur even when this was not appropriate. "Contralateral induction"' would have the effect of restoring appropriate localization of the signal.

The mislocalization effects found in the present paradigm would seem to fall within the same class phenomena as that of "contralateral induction," in that both effects appear to be based on mechanisms which, in everyday life, enable a more effective localization of signals emanating from multiple sources, yet can give rise to illusary mislocalizations in the laboratory.

\section{EXPERIMENT 2}

Experiment 1 compared two extreme conditions, the first where input to the two ears was strictly simultaneous and the second where these inputs were clearly separated in time. Experiment 2 explored the intermediate case; i.e., where input to the two ears overlapped but were not strictly simultaneous. This condition commonly occurs in normal listening, and also in experiments where natural speech is delivered "simultaneously" to both ears. It was expected that this intermediate case would yield results intermediate between the two extremes found in Experiment 1.

\section{Method}

\section{Procedure}

The procedure was identical to that in Experiment 1.

\section{Conditions}

The same two basic melodic patterns shown on Figures la and $1 \mathrm{~b}$ were employed. Sequences composed of these patterns were presented basically under four experimental conditions, the fourth consisting of four subconditions.

Conditions 1, 2, and 3. These conditions were identical to Conditions 1, 2, and 3 of Experiment 1.

Condition 4. This condition was identical to Condition 3, except that a 15-msec onset-offset asynchrony was incorporated between the melody component and the drone. The drone and melody component therefore always overlapped by $15 \mathrm{msec}$. In Condition $4 \mathrm{~L}$, the tone in the left ear always led the tone in the right ear, regardless of which ear received the melody component and which the drone. In Condition $4 \mathrm{R}$, the tone in the right ear always led the tone in the left ear. In Condition $4 \mathrm{M}$, the melody component always led the drone, regardless of whether it was presented to the left or the right ear. In Condition 4D, the drone always led the melody component.

Four examples of Pattern A and four of Pattern B were presented for each of Conditions 1,2, and 3, and also for each of the subconditions, $4 \mathrm{~L}, 4 \mathrm{R}, 4 \mathrm{M}$, and $4 \mathrm{D}$. In each case (with the exception of Condition 1 , where the melodies were binaurally presented), the distributions of the tones between the ears were identical to those in Conditions 2, 3, and 4 of Experiment 1.

The sequences were presented in random order in four blocks of 14 trials. The entire set of sequences was presented on two consecutive sessions, and the results were averaged. Subjects listened with earphones placed one way on the first session and reversed on the second session. The order of earphone placement was counterbalanced across subjects.

\section{Stimulus Parameters}

The stimulus parameters were identical to those in Experiment 1, except for Condition 4 , where the various asynchrony parameters led to differences in the durations of the pauses between tones within a sequence.

\section{Apparatus}

The apparatus were identical to those in Experiment 1, except that the stimuli were not recorded on tape, but were delivered on-line to the subjects in sound-insulated booths.

\section{Subjects}

Eleven undergraduates at the University of California at San Diego served as subjects in this experiment: they were chosen on the same criteria as for Experiment 1. The author also served as subject.

\section{Results}

The percentage correct recognitions of the melodic patterns in the different conditions of the experiment are shown on Table 2 . It can be seen that, in Conditions 1,2 , and 3 , essentially the same results were obtained as in Experiment 1 . Highly significant differences were found on comparing the condition where input to the two ears was nonsimultaneous, with the conditions where this input was simultaneous, either because the melody was presented binaurally, or because a drone was presented to the opposite ear 
Table 2

Percent Average Error in the Different Conditions of Experiment 2

\begin{tabular}{lr}
\hline \multicolumn{1}{c}{ Condition } & $\begin{array}{c}\text { Error } \\
\text { Rate }\end{array}$ \\
\hline $\begin{array}{l}\text { (1) Melody binaural; no drone. } \\
\text { (2) Component tones of melody switch between ears; } \\
\text { no drone. }\end{array}$ & 3.6 \\
$\begin{array}{l}\text { (3) Component tones of melody switch between ears; } \\
\text { drone presented in synchrony to opposite ear. }\end{array}$ & 37.0 \\
(4) Component tones of melody switch between ears; & 10.4 \\
$\begin{array}{l}\text { drone presented asynchronously to opposite ear. } \\
\text { (4L) Tone in left ear always leads tone in right. }\end{array}$ & 18.9 \\
(4R) Tone in right ear always leads tone in left. & 19.8 \\
(4M) Melody component always leads drone. & 21.4 \\
(4D) Drone always leads melody component. & 20.8 \\
\hline
\end{tabular}

(Condition 1 vs. Condition 2; Condition 3 vs. Condition $2 ; p<.01$, two-tailed, on Wilcoxon tests for both comparisons). The difference between Conditions 1 and 3 was marginally significant $(p=.05$, two-tailed, on a Wilcoxon test), and, given the results of Experiment 1, this may be attributed to the slight interference effect of the drone.

As expected, the overall performance level in Condition 4 , where the drone and melody component were asynchronous, fell between the levels in the strictly simultaneous and in the strictly nonsimultaneous conditions. Performance was significantly worse than in the simultaneous conditions (Condition 1 vs. Condition $4 ; \mathrm{p}<.01$, two-tailed; Condition 3 vs. Condition $4, p=.01$, two-tailed, on Wilcoxon tests for both comparisons). Yet, performance was significantly better than in the nonsimultaneous condition (Condition 2 vs. Condition 4, p $<.01$, twotailed, on a Wilcoxon test).

No significant differences were found on comparing the four subconditions, $4 \mathrm{~L}, 4 \mathrm{R}, 4 \mathrm{M}$, and $4 \mathrm{D}$. It may be noted, however, that the performance level in Condition 4D, where the drone led the melody, was higher than in the other three subconditions. Though this difference did not reach statistical significance, it might indicate a lag effect such as found in dichotic tasks involving speech materials (Berlin, Lowe-Bell, Cullen, Thompson, \& Loovis, 1973; Studdert-Kennedy, Shankweiler, \& Schulman, 1970).

\section{Discussion}

The finding that onset and offset asynchrony produces less integration of the input to the two ears indicates that such asynchrony results in an increased tendency to treat this input as emanating from different sources. This is as expected, since temporal similarities in the waveform envelopes of two simultaneous signals are important indicators that these signals are emanating from the same source, and discrepancies would indicate that they were emanating from different sources (Tobias, 1972).

In a related experiment, Bregman and Pinker (1978) presented a simultaneous two-tone complex in alternation with a third tone under various conditions of onset-offset asynchrony between the simultaneous tones. It was found that asynchrony increased the likelihood that one of the simultaneous tones would form a melodic stream with the third tone. The authors aruges that asynchrony between the simultaneous tones led to a decreased tendency for these tones to be treated as emanating from the same source, and so facilitated a sequential organization by frequency proximity between one of these simultaneous tones and the alternating tone.

Another related experiment is that of Rasch (1978). He studied the threshold for perception of a high tone when this was accompanied by a low tone. It was found that delaying the onset of the low tone relative to the high tone produced a substantial lowering of threshold: each $10 \mathrm{msec}$ of delay resulted in roughly a 10-dB downward shift in threshold, and at a delay of $30 \mathrm{msec}$ the threshold was close to that for the high tone in the quiet. Furthermore, under conditions of asynchrony, the subjective percept was strikingly changed, so that the two tones stood apart clearly rather than being fused into a single percept. The author interpreted these findings along the same lines as in the present paper.

Rasch also noted that despite its strong effects, the temporal asynchrony was not recognized as such by the subjects. The same observation was made in the present experiment. The subjective impression in the asynchronous conditions is that of a "plopping" sound at onset and offset of the two-tone complex, but it would be difficult to describe it subjectively in greater detail. Thus, the strong effect found here of temporal asynchrony on the formation of melodic configurations is not based on conscious inference.

The present experiments, apart from their general implications for theories of selective listening, are also of specific relevance to attentional mechanisms in music. Helmholtz (1859) early raised the question of how, given the complex, changing spectrum produced by a group of instruments playing simultaneously, we manage to reconstruct our musical environment so that certain components are fused to produce a single sound impression, and others are heard as separate melodic lines which are simultaneously perceived. In the latter case, the further question arises as to the basis on which such simultaneous melodic lines are constructed. For instance, he wrote:

Now there are many circumstances which assist us first in separating the musical tones arising from different sources, and secondly, in keeping together the partial tones of each separate source. Thus when one musical tone is heard for some time before being joined by the second, and then the second continues after the first has ceased, the separation in sound is facilitated by the succession of time. We have already heard the first musical tone by itself, and hence know immediately what we have to deduct from the compound effect for the effect of this first tone. Even when several parts pro- 
ceed in the same rhythm in polyphonic music, the mode in which the tones of different instruments and voices commence, the nature of their increase in force, the certainty with which they are held, and the manner in which they die off, are generally slightly different for each..... But besides all this, in good part music, especial care is taken to facilitate the separation of the parts by the ear. In polyphonic music proper, where each part has its own distinct melody, a principal means of clearly separating the progression of each part has always consisted in making them proceed in different rhythms and on different divisions of the bars (p. 59).

\section{And later:}

All these helps fail in the resolution of musical tones into their constituent partials. When a compound tone commences to sound, all its partial tones commence with the same comparative strength; when it swells, all of them generally swell uniformly; when it ceases, all cease simultaneously. Hence no opportunity is generally given for hearing them separately and independently (p. 60).

Recently, this issue has been raised in depth by Erickson (1975) exploring many musical examples. Although artificial stimulus conditions were employed in the present study, these results may well generalize to natural musical situations.

\section{REFERENCES}

Ahroon, W. A., Pastore, R. E., \& Wolz, J. P. Selective attention I. Two-channel simultaneous frequency difference limen. Journal of the Acoustical Society of America, 1977, 61, 811-815.

Axelrod, S., Guzy, L. T., \& Diamond, I. T. Perceived rate of monotonic and dichotically alternating clicks. Journal of the Acoustical Society of America, 1968, 43, 51-55.

BENADE, A. H. Fundamentals of musical acoustics. New York: Oxford University Press, 1976.

Berlin, C. I., Lowe-Bell, S. S., Cullen, J. K., Thompson, C. L., \& Loovis, C. F. Dichotic speech perception: An interpretation of right ear advantage and temporal offset effects. Journal of the Acoustical Society of A merica, 1973, 53, 699-709.

Bregman, A. S. The formation of auditory streams. In J. Requin (Ed.), Attention and performance VII. Hillsdale, N.J: Erlbaum, 1978.

Bregman, A. S., \& Camprell, J. Primary auditory stream segregation and perception of order in rapid sequences of tones. Journal of Experimental Psychology, 1971, 89, 244-249.

Bregman, A. S., \& Pinker, S. Auditory streaming and the building of timbre. Canadian Joumal of Psychology, 1978, 32, 20-31.

BroAdBENT, D. E. The role of auditory localization in attention and memory span. Journal of Experimental Psychology, 1954, 47, 191-196.

Broadennt, D. Perception and communication. London: Pergamon Press, 1958.

Cherry, E. C., \& Taylor, W. K. Some further experiments upon the recognition of speech, with one and with two ears. Journal of the Acoustical Society of America, 1954, 26, 554-559.

DEUTSCH, D. Two-channel listening to musical scales. Journal of the Acoustical Society of America, 1975, 57, 1156-1160. (a)

DeutsCh, D. Musical illusions. Scientific American, 1975, 233. 92-104. (b)
Dowling, W. J. The perception of interleaved melodies. Cognitive Psychology, 1973, 5, 322-337.

Egan, J. P., \& Benson, W. Lateralization of a weak signal presented with correlated and with uncorrelated noise. Journal of the Acoustical Society of America, 1966, 40, 20-26.

Erickson, R. Sound structure in music. Berkeley: University of California Press, 1975.

Ha as, H. Über den einfluss eines Einfachechos auf die Hörsamkeit von Sprache. Acustica, 1951, 1, 49-52.

HeLmholtz, H. L. On the sensations of tone as a physiological basis for the theory of music (2nd English ed.). New York: Dover, 1954. (Originally published 1859.)

Huggins, A. W. F. Distortion of the temporal pattern of speech: Interruption and alternation. Journal of the Acoustical Society of America, 1964, 36, 1055-1064.

Judd, T. Comments on Deutsch's musical scale illusion. Perception \& Psychophysics, in press.

Moray, N. A data base for theories of selective listening. In P. M. A. Rabbitt \& S. Dornic (Eds.), Attention and performance V. London: Academic Press, 1975.

Pollack, I. Temporal switching between binaural information sources. Journal of the Acoustical Society of America, 1978, $63,550-558$.

Puleo, J. S., \& Pastore, R. F. Critical band effects in twochannel auditory signal detection. Journal of Experimental Psychology: Human Perception and Performance, in press.

Rasch, R. A. The perception of simultaneous notes such as polyphonic music. Acustica, 1978, 40, 1-72.

Schubert, E. D., \& Parker, C. D. Addition to Cherry's findings on switching speech between the two ears. Journal of the Acoustical Society of America, 1956, 27, 792-794.

Shiffrin, R. M., Pisoni, D. B., \& Castaneda-Mendez, K Is attention shared between the ears? Cognitive Psychology, $1974,6,190-215$.

Sorkin, R. D., Pastore, R. E., \& Pohlmann, L. D. Simultaneous two-channel signal detection. II. Correlated and uncorrelated signals. Joumal of the Acoustical Society of America, 1972, 51, 1960-1965.

Sorkin, R. D., Pohlmann, L. D., \& Gilliom, J. D. Simultaneous two-channel signal detection. III. 630 - and $1400-\mathrm{Hz}$ signals. Journal of the Acoustical Society of America, 1973, 14, 101-109. Studdert-Kennedy, M., Shankweiler, D., \& Schulman, S. Opposed effects of a delayed channel on perception of dichotically and monotonically presented $\mathrm{CV}$ syllables. Journal of the Acoustical Society of A merica, 1970, 48, 599-602.

Tobias, J. V. Curious binaural phenomena. In J. V. Tobias (Ed.), Foundations of modern auditory theory (Vol. II). New York: Academic Press, 1972.

Tobias, J. V., \& Schubert, E. D. Effective onset duration of auditory stimuli. Journal of the Acoustical Society of America, $1959,31,1595-1605$

Treisman, A. M. Shifting attention between the ears. Quarterly Journal of Experimental Psychology, 1971, 23, 157-167.

VAN NoORDEN, L. P. A. S. Temporal coherence in the perception of tone sequences. Unpublished doctoral dissertation, Technische Hogeschoel Eindhoven, Eindhoven, The Netherlands, 1975.

Wallach, H., Newman, E. B., \& Rosenzeig, M. R. The precedence effect in sound localization. American Journal of Psychology, 1949, 62, 315-336.

WARREN, R. M., \& BAShFord, J. A. Auditory contralateral induction: An early stage in binaural processing. Perception \& Psychophysics, 1976, 20, 380-386.

(Received for publication September 11, 1978; revision accepted February 9, 1979.) 